Copyright (C) 2005 IEEE. Reprinted from

IEEE Transactions on Magnetics, 2005; 41 (10):3826-3828

This material is posted here with permission of the IEEE. Such permission of the IEEE does not in any way imply IEEE endorsement of any of the University of Adelaide's products or services. Internal or personal use of this material is permitted. However, permission to reprint/republish this material for advertising or promotional purposes or for creating new collective works for resale or redistribution must be obtained from the IEEE by writing to pubs-permissions@ieee.org.

By choosing to view this document, you agree to all provisions of the copyright laws protecting it. 


\title{
A Multipole Array Magnetic Spring
}

\author{
Will Robertson, Ben Cazzolato, and Anthony Zander
}

Active Noise and Vibration Control Group, School of Mechanical Engineering, University of Adelaide, SA, Australia 5005

This paper presents research on a magnetic spring concept, which has application to the development of a vibration isolation table. Features of the design are scalable, noncontact load bearing and a single degree of instability.

Index Terms-Magnetic forces, magnetic levitation, magnetic spring, multipole arrays, permanent magnetics.

\section{INTRODUCTION}

$\mathbf{V}$ IBRATION isolation is a requirement for a variety of sensitive equipment, as undesirable vibrations can often cause inaccuracy or error. Commercial vibration isolation tables for cleanroom environments typically use pneumatic springs. It is proposed here that noncontact magnetic springs could be used instead, eliminating the path of physical vibration transmission.

The prior art for levitation tables show designs that are effective vibration isolators, but not well suited for efficient large load bearing. For example, the design of Mizuno et al. [1] is unstable in the vertical direction, and that of Choi et al. [2] uses singular magnets which do not scale well with volume.

This paper outlines the design of a noncontact magnetic arrangement that is able to passively bear large loads and is also potentially easily integrated into a control system to remove the inevitable instability elucidated by Tonks [3].

\section{BASIC MAgnetic SpRING Design}

Two magnets in repulsion create a passive spring force between them. In a vertical arrangement with the lower magnet fixed, the floating magnet is unstable in both horizontal directions. A more stable arrangement is to use three magnets arranged horizontally in attraction, with the outer two fixed and the floating inner magnet acting as the spring element (this is similar in behavior to an axial magnetic bearing). The floating magnet is stable in the vertical direction and also in the direction perpendicular to the position of the fixed magnets. Diagrams of these arrangements are shown using cube magnets in Fig. 1.

The more stable horizontal spring is, however, less appropriate for supporting weight. Graphs of force versus vertical displacement for these arrangements, solved using Bancel's analytical "magnetic nodes" technique [4] for 15-mm cube magnets, are shown in Fig. 2. Here, the rest position for the vertical spring is 1.5 magnet widths and the gap between the horizontally arranged magnets is $0.1,0.5$, and 1 magnet widths in three separate cases. The weight of the magnets due to gravity is neglected. It can be seen that with the exception of very close horizontal magnet arrangements, the vertical spring may support much greater loads with an exponentially increasing stiffness; furthermore, the horizontal spring becomes unstable if the vertical load is increased past the peak force.

Digital Object Identifier 10.1109/TMAG.2005.854981

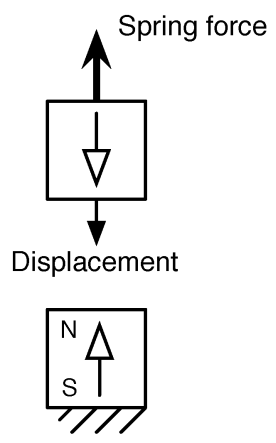

Vertical Spring

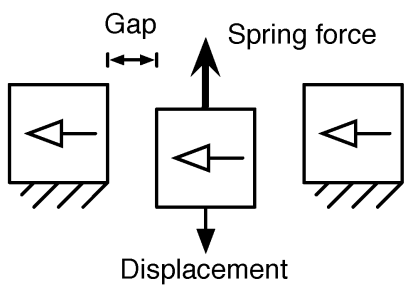

Horizontal Spring
Fig. 1. Schematic of two simple magnetic springs: vertical arrangement in repulsion and a horizontal arrangement in attraction.

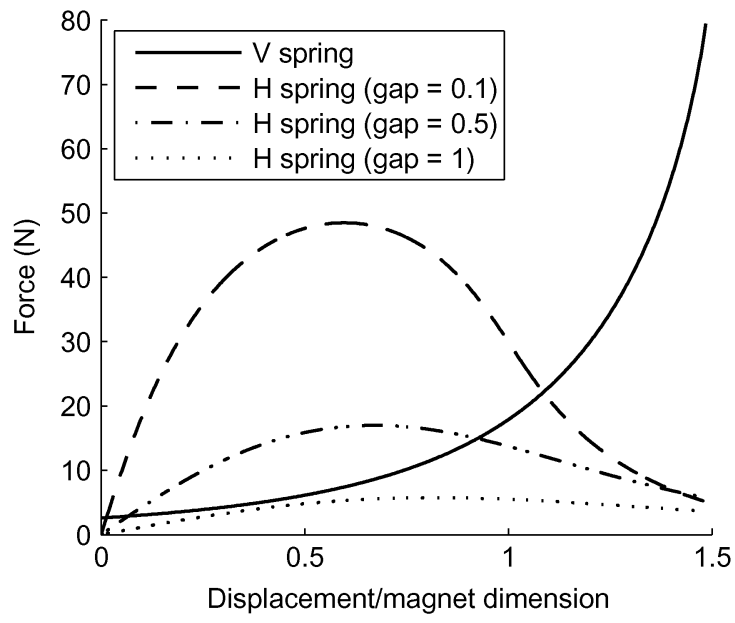

Fig. 2. Force versus displacement curves for the arrangements shown in Fig. 1 for $15-\mathrm{mm}$ cube neodymium magnets. Gap is normalized by the magnet dimension.

A combination of the vertical spring and the horizontal spring yields a system that is capable of supporting large loads in the vertical direction and is unstable in only a single horizontal direction. This instability may be constrained with passive guides or an active control system with noncontact electromagnetic actuators.

\section{Multipole ARRAYS FOR StRENGTH}

This "combination spring" design may be improved by replacing the homogeneous magnets with multipole arrays. Such arrays have been used in magnetic bearings for increased stiffness [5] and their application here follows similar principles. 


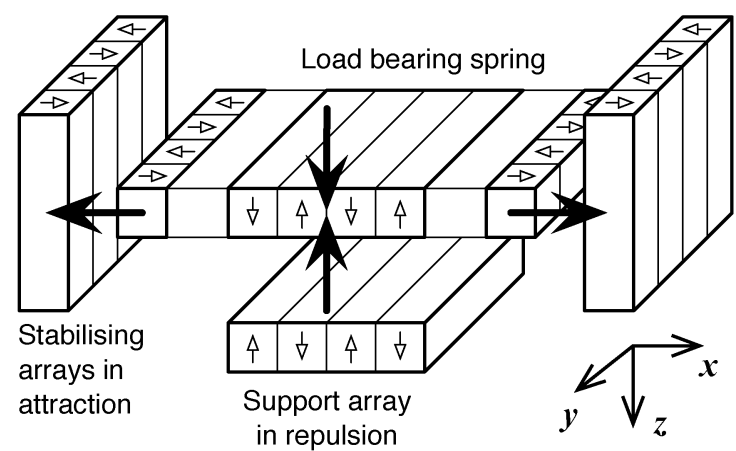

Fig. 3. Complete magnetic spring design with multipole arrays. Solid arrows show the $x$ and $z$ reaction forces on the spring.

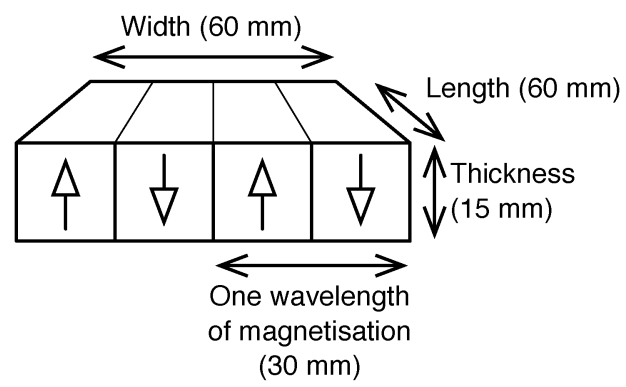

Fig. 4. Geometry of the individual stabilizing, support, and load bearing arrays. Wavelength of magnetization of the multipole arrays is twice their thickness.

Fig. 3 shows the design of a combination spring that suits the original requirements for stability and load bearing. The fixed horizontal arrays stabilize the spring in the $y$ direction even over a range of vertical displacements corresponding to large variations in the load on the spring.

To demonstrate the concept, 15 -mm-thick neodymium magnets are used in a magnetic nodes analysis of the forces on the spring due to displacements in every direction. Each array contains four magnets alternating in magnetization as shown in Fig. 4. The gaps between the fixed and floating arrays at rest are all equal to the array thicknesses.

\section{A. Load Bearing}

At rest, the supporting force of this spring is approximately $70 \mathrm{~N}$. Halving the gap increases this value fourfold (see the $z$ direction curve of Fig. 7 later). Increasing the load bearing capacity may be achieved by simply increasing the length and width of the arrays by adding more magnets. The use of multipole arrays provides this scalability without the need to increase the thickness of the arrays.

The magnetic nodes technique restricts the multipole arrays used in this analysis to two magnets per polarization wavelength, with $180^{\circ}$ rotations of magnetization between successive magnets. Stronger forces than reported here are possible by using $90^{\circ}$ magnetization rotations with four half-width magnets or, even better, $45^{\circ}$ magnetization rotations with eight quarter-width magnets, in order to accurately approximate sinusoidal magnetization [6].

A further advantage of using more divisions per wavelength is the focusing effect on the magnetic flux: in the ideal case of

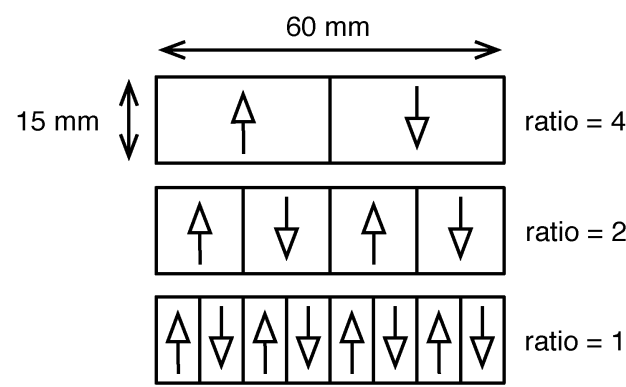

Fig. 5. Cross sections of the first three variations of magnetization wavelength to thickness ratio for the curves shown in Fig. 6.

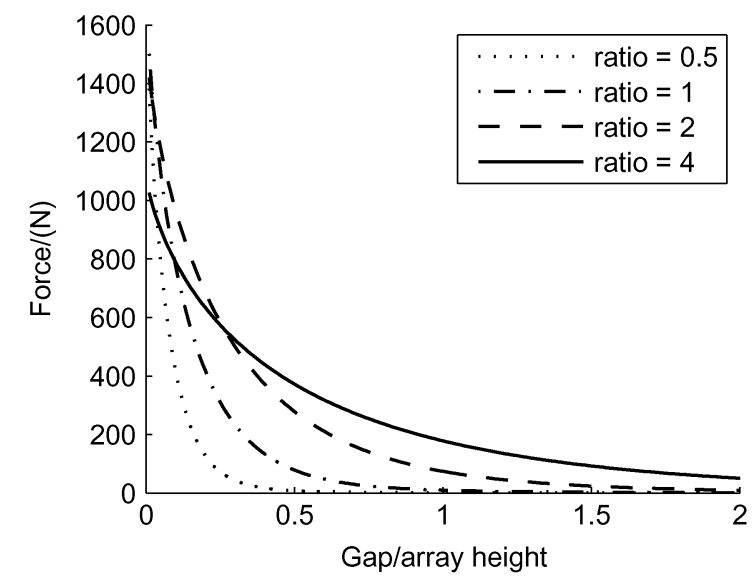

Fig. 6. Forces between opposing multipole arrays for varying ratios of wavelength of magnetization to array thickness, as shown in Fig. 5.

sinusoidal magnetization, the flux is entirely single-sided; but with just two magnets per wavelength, the magnetic field is still symmetrical. The absence of leakage flux in the former case removes the possibility of side-effects due to its interaction with other parts of the device.

Another matter to consider is the wavelength of magnetization. Fig. 5 shows three ratios of magnetization wavelength to array thickness. As this ratio decreases, the gradient of the force (that is, the stiffness) between two facing arrays increases while acting over increasingly smaller distances. This is shown in Fig. 6 for four pairs of square arrays in opposition. A good compromise between spring stiffness and range can be achieved with a wavelength of twice the array thickness: this ensures a sufficient gap for a range of displacement while still providing a reasonable stiffness.

This result is consistent with the analysis of a multipole array levitating against a superconductor [7]. However, there is a notable difference between the behavior of a multipole array levitating against a superconductor and the behavior of two opposing multipole arrays.

In the former case, the levitation force is created between the magnet array and a mirror of itself in the superconducting material. Thus, any horizontal shifts of the magnet array are mirrored in the superconductor and the levitation force remains constant. This is a great advantage when using superconductive levitation, but, unfortunately, the inconvenience and cost of using such material outweighs its advantage for many applications. 


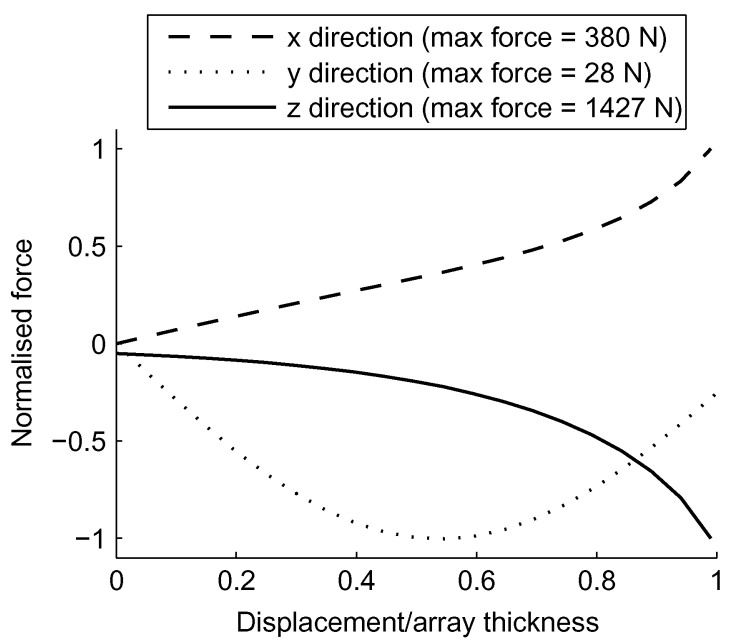

Fig. 7. Forces on the spring in the direction of displacements in each axis. Curves are normalized by their respective peak values (shown in the legend) to display their shape.

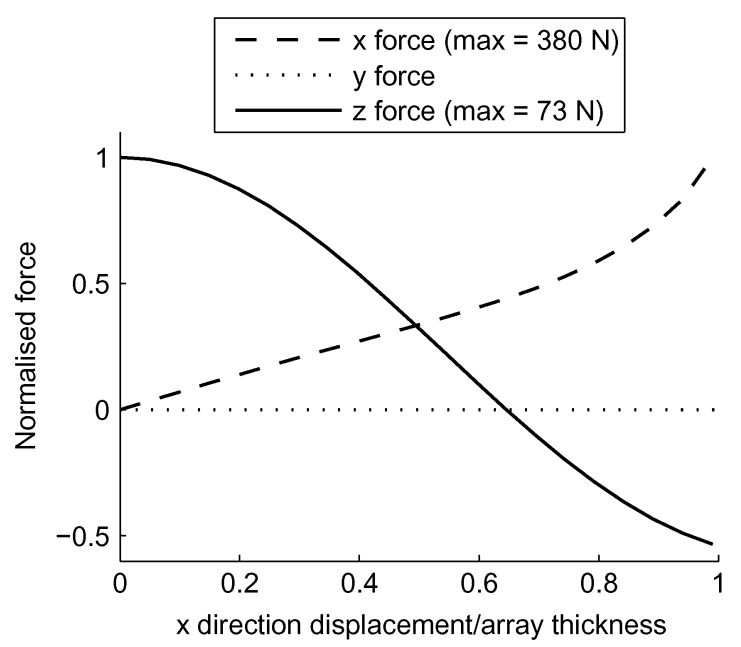

Fig. 8. Normalized forces on the spring in each direction for displacements in the $x$ direction.

In the case with two repulsive magnet arrays, lateral displacements affect the levitation force: a half-wavelength displacement will result in attraction between the arrays, compromising the stability of the system (this will be seen in the next section in Fig. 8).

\section{B. Stability of the Multipole Spring}

Fig. 7 shows the forces on the spring due to displacements in each direction. Stable motion requires a negative gradient on these curves, since in this case the reaction force will be in opposition to the displacement. In the centred position, the spring is in unstable equilibrium with instability in the horizontal $x$ direction only. This instability force is quite linear over most of the displacement, which is convenient for control system design.

The use of multipole arrays limits the displacement of the spring in the horizontal directions, as demonstrated in the $y$ direction curve in Fig. 7. The stability reverses after a displacement of a quarter-wavelength of magnetization as the force-displacement gradient turns positive.

Further disincentive for horizontal motion is the reduction of vertical load bearing as mentioned in the previous section. In Fig. 8, the spring is displaced in the $x$ direction and the graph shows the resultant forces on the spring in each direction. This displacement causes an attenuation of the supporting force in the vertical $z$ direction. Instability occurs as the vertical force turns negative after a displacement of approximately one-third of a wavelength. This is greater than the displacement for instability in the $y$ direction because the stabilizing arrays also provide some supporting force.

\section{CONCLUSION}

The design of a large load bearing magnetic spring has been outlined and shown to have a single degree of instability in its centred position. Significant displacements yield further instability, so the spring must be constrained either by passive guides or an active control system. In this state, the spring may be used as the main support for a vibration isolation table. This latter application is the focus of ongoing research, the results of which will be reported at a later date.

\section{REFERENCES}

[1] T. Mizuno, H. Suzuki, M. Takasaki, and Y. Ishino, "Development of a three-axis active vibration isolation system using zero-power magnetic suspension," in Proc. 42nd IEEE Conf. Decision Control, Maui, HI, Dec. 2003, pp. 4493-4498.

[2] K.-B. Choi, Y. G. Cho, T. Shinshi, and A. Shimokohbe, "Stabilization of one degree-of-freedom control type levitation table with permanent magnet repulsive forces," Mechatronics, vol. 13, no. 6, pp. 587-603, Jul. 2003.

[3] L. Tonks, "Note on Earnshaw's theorem," Electr. Eng., vol. 59, no. 3, pp. 118-119, Mar. 1940.

[4] F. Bancel, "Magnetic nodes," J. Phys. D, Appl. Phys., vol. 32, pp. 2155-2161, 1999.

[5] B. Paden, N. Groom, and J. F. Antaki, "Design formulas for permanentmagnet bearings," Trans. ASME, vol. 125, pp. 734-738, Dec. 2003.

[6] K. Halbach, "Design of permanent multipole magnets with oriented rare earth cobalt material," Nucl. Instrum. Methods, vol. 169, no. 1, pp. 1-10, 1980.

[7] A. M. Campbell, "Forces between arrays of magnets and superconductors," Supercond. Sci. Technol., vol. 15, pp. 759-762, 2002.

Manuscript received February 3, 2005 